\title{
Development of junction temperature estimation system for light-emitting LED using pulsed-laser Raman scattering
}

\author{
Makoto Horiuchi ${ }^{1}$, Yukihiko Yamagata ${ }^{2 *}$, Shin-ichi Tsutsumi ${ }^{2}$, Kentaro Tomita ${ }^{2}$ and Yoshio Manabe ${ }^{1}$
}

\author{
* Correspondence: \\ yamagata@asem.kyushu-u.ac.jp \\ Thematic Series on [14th \\ International Symposium on the \\ Science and Technology of Lighting \\ (LS14)] \\ ${ }^{2}$ Interdisciplinary Graduate School \\ of Engineering Sciences, Kyushu \\ University, 6-1 Kasugakoen, Kasuga \\ City, Fukuoka 816-8580, Japan \\ Full list of author information is \\ available at the end of the article
}

\begin{abstract}
A new method by using a pulsed-laser Raman scattering technique was employed to estimate junction temperature of a fluorescence-resin-less blue-LED package. Temperature dependent Raman shift of $E_{2}{ }^{H}$ mode of GaN layer in the blue-LED were obtained and were in good agreement with the experimental data published by the other researchers. This technique was applied to estimation of a junction temperature of the light-emitting blue-LED. The junction temperature was successfully estimated even under the strong stray light and LED light emission. It is considered that the proposed technique would be a remote and standard system for measuring the junction temperature of light-emitting white-LEDs.
\end{abstract}

Keywords: GaN; LED; Raman spectroscopy; Pulsed-laser; Junction temperature

\section{Background}

Recently, III-V nitride semiconductors having a relatively large band gap have been widely investigated for solid state blue and/or ultraviolet light sources. For these applications to light emitting devices (LEDs) and also other semiconductor power devices in commercial field, especially to a LED package for illumination application, it is required to integrate many chips on a package and to operate these under higher current. Efficiency, lifetime, and operational stability of these devices degrade remarkably resulting from the temperature rise of the junction of these devices. Thus the junction temperature is a critical parameter and strongly affects the efficiency, the output power, the life time and the reliability of these devices. Therefore, in order to fabricate a high quality device, junction temperature estimation of each LED chip on the device during the operation, and the heat radiation design of the whole device based on the experimental results are primary important.

There are some investigations in which the junction temperature of LEDs and/or laser diodes is estimated using micro-Raman spectroscopy $[2,5-7,10]$, temperature coefficient of diode-forward voltage $\left(V_{\mathrm{f}}\right)$ [13, 14], infrared imaging [9], electroluminescence [12], thermal resistance [8], and nematic liquid crystal thermographic technique [4]. However, the junction temperature of each chip on a LED package for illumination application, in which there are many chips electrically connected in series/parallel, is hardly obtained by $V_{\mathrm{f}}$ method where total current and total voltage are measured. Even if all chips on the

(c) 2015 Horiuchi et al. This is an Open Access article distributed under the terms of the Creative Commons Attribution License (http:// creativecommons.org/licenses/by/4.0), which permits unrestricted use, distribution, and reproduction in any medium, provided the original work is properly credited. 
package are the same characteristics, a problem of the temperature distribution due to heat radiation characteristics of the package still remains. Although infrared imaging has a good advantage of 2D mapping of the temperature [9], proper setting of spectral emissivity coefficient and disability to a white-LED of which a phosphor- and scatterer-mixed resin is directly deposited are the issues which should be resolved. Micro-Raman scattering having very good spatial resolution has been widely applied to measure the junction temperature. However, because of very short working distance, application to simultaneous multipoint measurement for relatively large and multi-chip white-LED package is difficult. Thus, in our knowledge, estimation of the junction temperature of operating white-LED package for illumination application has not been performed, although it is strongly required to develop the estimation system.

In this research, it is aimed to develop a pulsed-laser Raman scattering method as a remote and standard system for estimation of the junction temperature of LEDs including white-LED during the operation. Using a nanosecond pulsed-laser as an incident light source, sufficient laser power density for detection of weak Raman signal can be achieved even at relatively large irradiation area of a LED package placed far from the laser injection and scattering light detection optics, instead of a CW laser being focused to very small area. A pulsed-laser Raman scattering technique, which is equipped with ns-order pulsed-laser and long focusing lens, has potentials of remote measurement and simultaneous multipoint measurement, which lead to 2D mapping of the temperature of the LED package in the future. Also, as influence of strong disturbance light such as LED emission itself can be eliminated by a combination with a highly time-resolved detection system, for example a gated-ICCD camera, so the measurement for LED projector lamp, for example, would be enabled. Therefore, this technique is considered to be one of the prospective candidates of temperature estimation method of white LEDs for illumination application.

In this paper, observation of Raman spectra from a fluorescence-resin-less blue-LED chip by a pulsed-laser Raman scattering method is presented. Dependence of the LED temperature on the Raman shift of $E_{2}{ }^{\mathrm{H}}$ mode of GaN layer and an estimation of the junction temperature of the fluorescence-resin-less blue-LED chip by the Raman shift are described.

\section{Methods}

Experimental setup for measuring junction temperature of LED using a pulsed-laser Raman scattering system which is developed at this research is schematically illustrated in Fig. 1. A dye laser (Continuum ND6000) was pumped by SHG light of a Nd:YAG laser (Continuum PL8000) for the generation of $632.816 \mathrm{~nm}$ laser light at repetition rate of $10 \mathrm{~Hz}$. In order to remove amplified spontaneous emission included in the dye laser beam, a laser line filter (Edmund, 68-879, FWHM: 3 nm, O.D. >4) was used. The laser beam that passed a linear polarization plate was focused on to one of the chips on a LED package by a spherical lens $(f=100 \mathrm{~mm})$.

A fluorescence-resin-less blue-LED package used in this study was placed on an electric heater block, as shown in Fig. 2a. The temperature of whole of the LED package was changed from room temperature to $180^{\circ} \mathrm{C}$ by PID control using the electric heater and a K-type thermocouple $\left(\begin{array}{lll}\phi & 1 & \mathrm{~mm}\end{array}\right)$ which was inserted between the LED and the heater block. The LED of which the temperature was kept constant was irradiated by the laser 


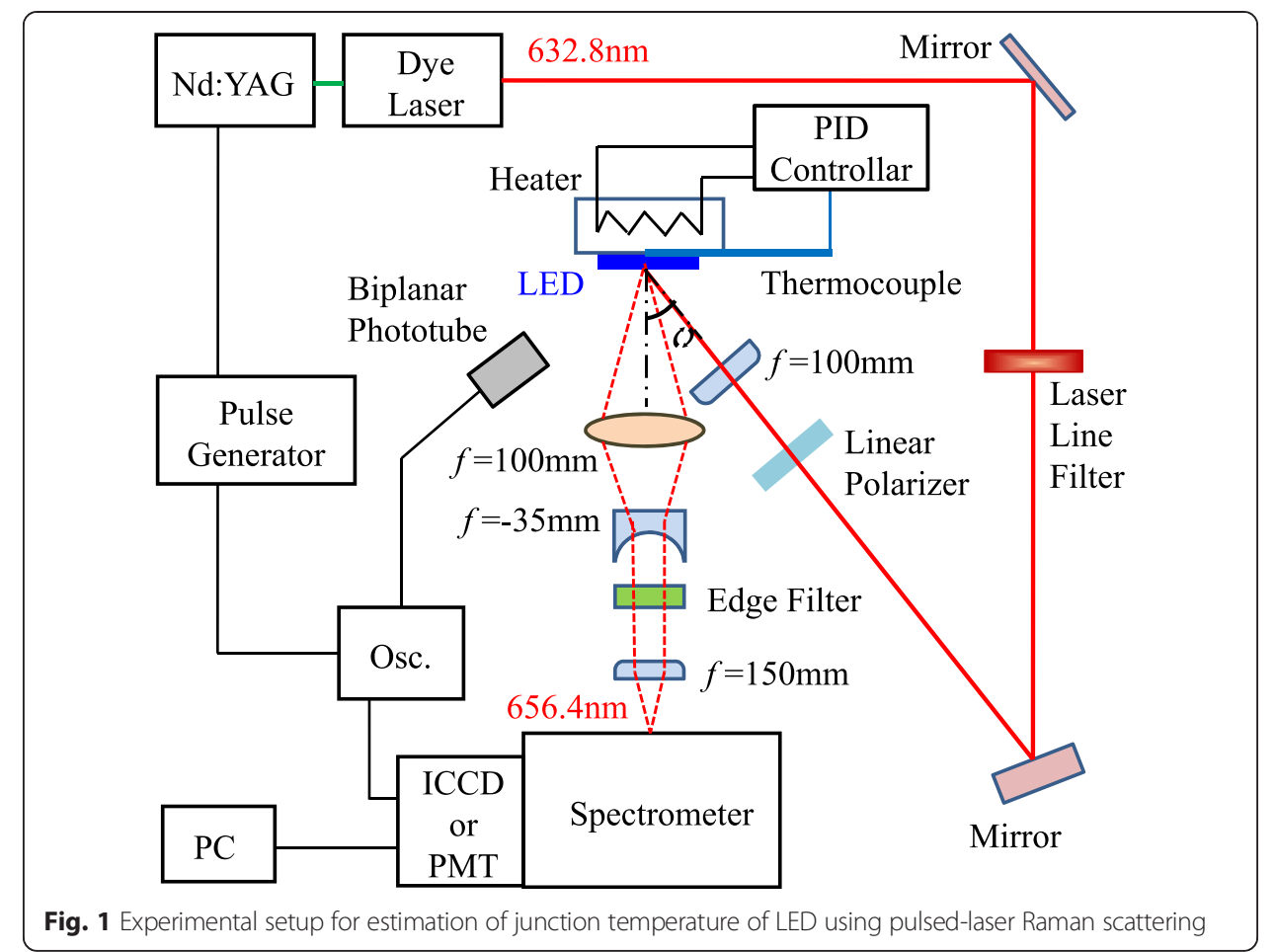

beam with an incident angle of $22-60^{\circ}$. The laser energy, the laser pulse duration, and the laser irradiated area of the LED surface was $0.12 \mathrm{~mJ}, 10 \mathrm{~ns}, \phi 1.0 \mathrm{~mm}$ (for $22^{\circ}$ case), respectively, therefore, the laser power density of about $1.5 \times 10^{10} \mathrm{~W} / \mathrm{m}^{2}$ was used for detection of Raman scattering signal. This value is comparable to micro-Raman scattering techniques. A part of the scattered light was imaged onto the entrance slit of a single spectrometer $(0.7 \mathrm{~m}, 1800$ grooves $/ \mathrm{mm}, \mathrm{F} 14)$ from passing through a collection lens $(f=100 \mathrm{~mm})$, a relay lens $(f=-35 \mathrm{~mm})$ and a Raman edge filter (Edmund, 86-243, $632.8 \mathrm{~nm}$, O.D. $>6)$ that cuts a very strong laser light.

The Raman scattering spectra (near 656.4 nm) of $E_{2}{ }^{\mathrm{H}}$ mode of GaN layer in the blueLED were detected by a photomultiplier tube (Hamamatsu, R1333) or a gated-ICCD camera (Princeton Instruments, PI-MAX/UNIGEN II) which were equipped with the

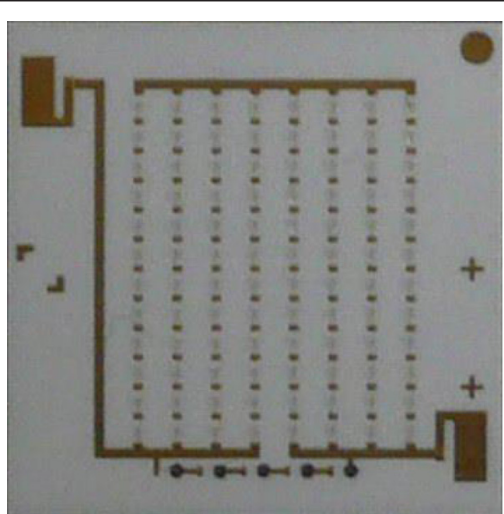

(a)

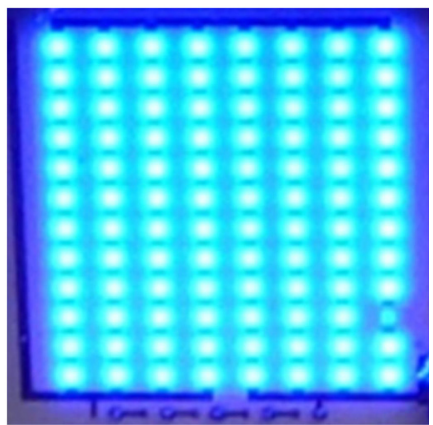

(b)

Fig. 2 Photographs of $\mathbf{a}$ a measured blue-LED package and $\mathbf{b}$ the light-emitting LED 
spectrometer. In order to observe the weak Raman scattering signal, the signal was accumulated for up to 20,000 laser shots with the photon counting technique in both cases of the PMT and the gated-ICCD camera. First, Raman spectra of the GaN- $E_{2}{ }^{\mathrm{H}}$ at various temperatures, where the LED was not operating (no emission light), were observed to realize temperature dependence of the Raman shift of the $\mathrm{GaN}-E_{2}{ }^{\mathrm{H}}$ from one chip on the fluorescence-resin-less blue-LED package. Then an estimation of the junction temperature of one chip located at the center of the blue-LED package during the operation (Fig. 2b) was demonstrated using the temperature dependence of Raman shift of the $\mathrm{GaN}-E_{2}{ }^{\mathrm{H}}$. Furthermore, the influence of a phosphor- and scatterer-mixed resin of a white-LED, which is deposited on the surface of the blue-LED package, on the Raman scattering measurement was investigated. Through these investigations, the usefulness and the possibility of the proposed technique as the junction temperature estimation method of a white-LED package during the operation is evaluated.

\section{Results and discussion}

Raman scattering signal from the GaN layer of the measured LED package was decreased much with increasing of the laser incident angle, all of experiment below was done at the laser incident angle of $22^{\circ}$. Figure 3 shows Raman spectra (Stokes line) of the GaN- $E_{2}{ }^{\mathrm{H}}$ observed at various temperatures, where the fluorescence-resin-less blue-LED was not operating. The vertical axis represents the signal intensity accumulated for 20,000 laser shots by the photon counting mode of the gated-ICCD. The horizontal axis represents the relative shift of the $\mathrm{GaN}-E_{2}{ }^{\mathrm{H}}$ Raman peak from the one at $0 \mathrm{~K}$, in which the peak shift at the room temperature $\left(20^{\circ} \mathrm{C}\right)$ is obtained of $66.5 \mathrm{~nm}$ by Song et al. [11]. As can be seen in Fig. 3, a shift to the short wavelength side of the Raman peak and a decrease of signal intensity are observed along with a temperature increase of the LED. The temperature dependence of the Raman shift of the $\mathrm{GaN}-E_{2}{ }^{\mathrm{H}}$ mode, which is obtained from both measurements using the PMT and the gated-ICCD, is shown in Fig. 4. In the Fig. 4, the experimental curve obtained by Liu et al. using micro-Raman spectroscopy [7] is also

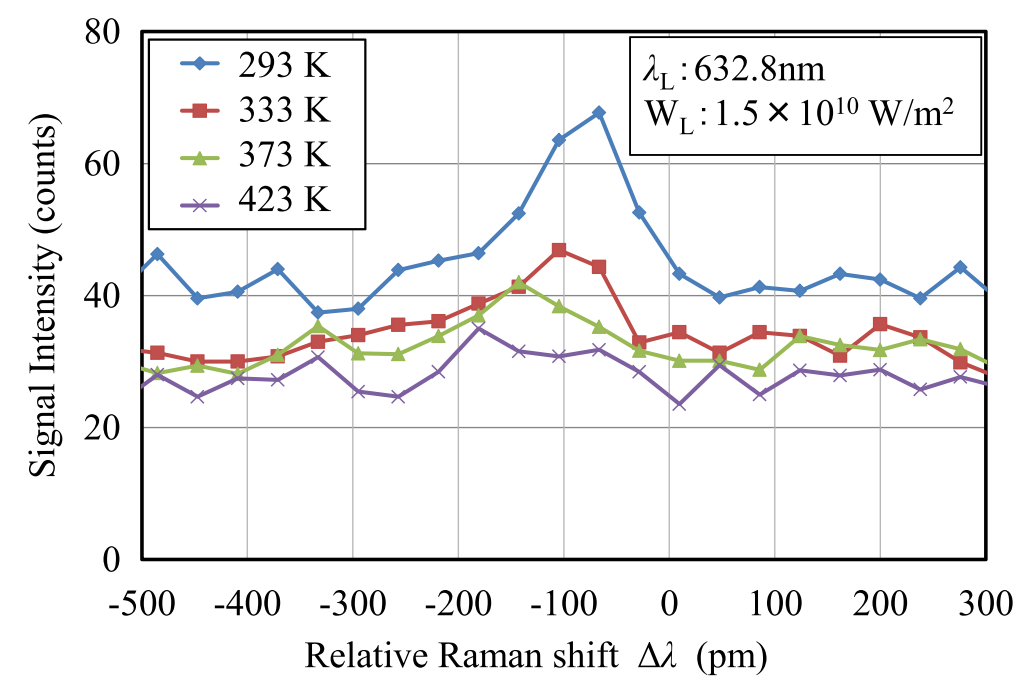

Fig. 3 Raman spectra of the $\mathrm{GaN}-\mathrm{E}_{2}{ }^{\mathrm{H}}$ from one chip on a fluorescence-resin-less blue-LED package observed at various temperatures. Temperature of the blue-LED was kept constant and the LED was not operating (no emission light) 


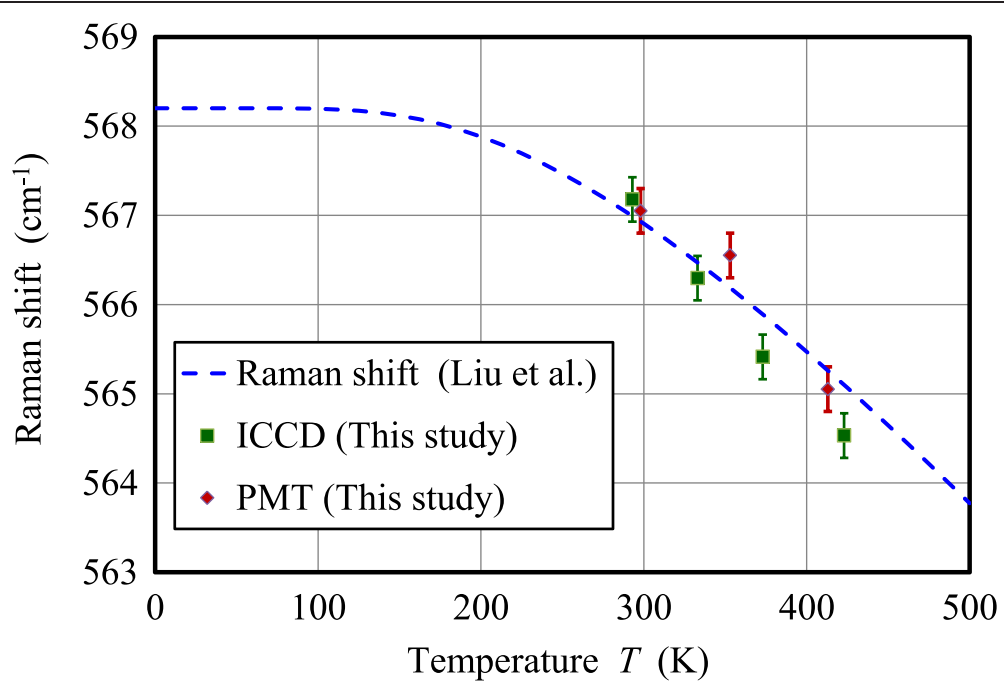

Fig. 4 Temperature dependence of the Raman shift of the $\mathrm{GaN}_{\mathrm{N}} \mathrm{E}_{2}{ }^{\mathrm{H}}$ of the blue-LED. The broken line shows the fitting curve of experimental data obtained by Liu et al. [1]

shown. The function form of the temperature dependence of the Raman shift in GaN, which is given by Liu et al., is as follows;

$$
\omega(T)=\omega_{0}-\frac{A}{\exp \left(B h c \omega_{0} / \kappa_{B} T\right)-1},
$$

where $\omega_{0}$ is the Raman phonon frequency at $0 \mathrm{~K}$, and $A$ and $B$ are fitting parameters [7]. For the $E_{2}{ }^{\mathrm{H}}$ mode, they give $\omega_{0}=568.2 \pm 0.2 \mathrm{~cm}^{-1}, A=17.9 \pm 2.4 \mathrm{~cm}^{-1}$, and $B=0.99 \pm$ 0.08 , where the experimental data obtained by them in the temperature range of $90-$ $770 \mathrm{~K}$ fit this form well. As can be seen in Fig. 4, the Raman shifts obtained by this method are almost in accord with the curve within slight differences. The Raman shift is affected by the internal stress depending on the under and/or the upper layer of GaN layer $[7,11]$. Although the slight difference seems to be caused by the internal stress or the temperature difference between the $\mathrm{GaN}$ layer and the thermocouple contacting point, it is not well clarified yet.

The pulsed-laser Raman scattering method was applied to estimation of the junction temperature of a fluorescence-resin-less blue-LED package during the operation. The junction temperature can be estimated not only the Raman shift but also the intensity ratio of stokes and anti-stokes of peak [2]. In this study, due to the functional limitation of the optics, the junction temperature estimation was made only from the shift of Raman peak by using the formula (Eq.1). The photograph of the LED operated at $50 \mathrm{~V}$ is shown in Fig. 2b. One of the chips of the blue-LED package was irradiated by the probe laser beam to measure the Raman spectra. The observed Raman spectra of the GaN- $E_{2}{ }^{\mathrm{H}}$ are shown in Fig. 5, in which the one observed at room temperature $\left(25^{\circ} \mathrm{C}\right)$ without operating is also shown. The other experimental conditions were the same as in the case of the measurement of Fig. 3. As can be seen in Fig. 5, apparent Raman spectra are observed even under the strong blue LED light, although the background of the spectra is relatively larger than that of Fig. 3. Using the temperature dependence of Raman shift of the GaN- $E_{2}{ }^{\mathrm{H}}$ (formula (Eq.1)), the junction temperature of the LED in case of $50 \mathrm{~V}$ and $55 \mathrm{~V}$ is estimated to be $100 \pm 20{ }^{\circ} \mathrm{C}$ and $190 \pm 20^{\circ} \mathrm{C}$, respectively. 


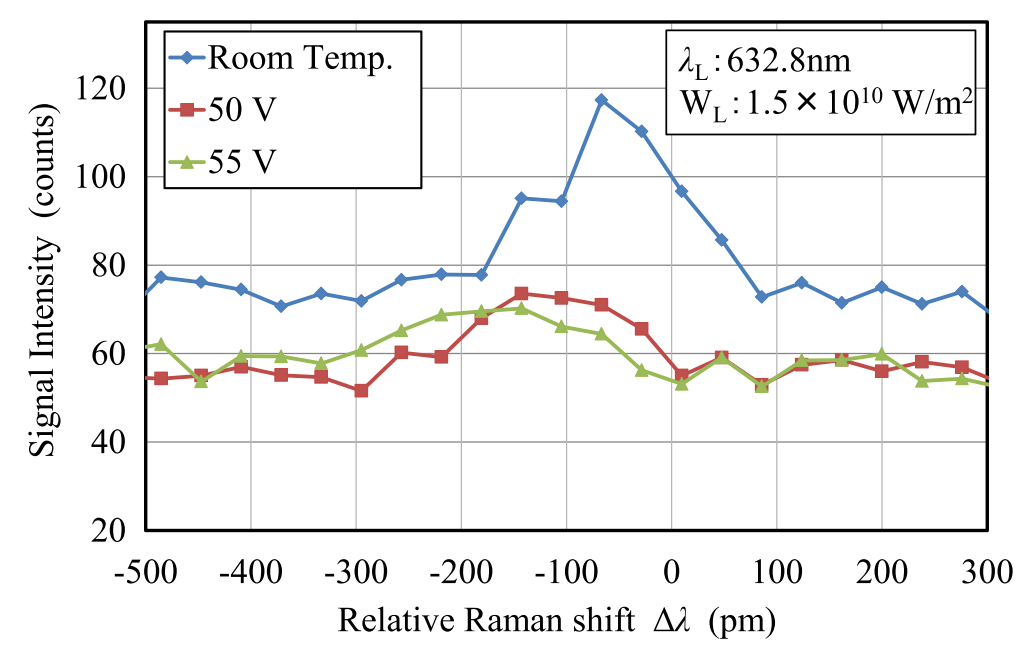

Fig. 5 Raman spectra of the $\mathrm{GaN}_{\mathrm{N}} \mathrm{E}_{2}{ }^{\mathrm{H}}$ observed at various operating voltages. The LED operating voltages are of $50 \mathrm{~V}$ and $55 \mathrm{~V}$. Raman spectrum from the same LED without operation at room temperature is also shown

In this experiment, the uncertainty of Raman shift measurement of about $30 \mathrm{pm}$ $\left(0.67 \mathrm{~cm}^{-1}\right)$ was caused mainly by instrumental function of the spectrometer and linewidth of the Raman peak itself. The accuracy of temperature estimation $( \pm 20 \mathrm{~K})$ was obtained from temperature gradient $66 \mathrm{~K} / \mathrm{cm}^{-1}$ of the Raman shift at $300-450 \mathrm{~K}$, which was calculated by using the formula (1).

On the other hand, detection of the Raman scattering signal from a white-LED is difficult due to a phosphor- and scatterer-mixed resin which is deposited directly on the surface of a bare blue-LED. There are two problems caused by the resin mixture. One is the fluorescence emitted from the phosphor by the laser excitation. In previous measurement [3] using the SHG of a Nd:YAG laser (SHG/YAG), large background noise was detected, because the laser wavelength of $532 \mathrm{~nm}$ and the Raman wavelength of $549 \mathrm{~nm}$ is in the absorption tail and in the fluorescence regime of the resin, respectively. In this study, we confirmed that the laser wavelength of $632.8 \mathrm{~nm}$ and the Raman wavelength near $656.4 \mathrm{~nm}$ is far from the absorption and fluorescence regimes of the phosphor, respectively. As the result, the absorption and the fluorescence by the phosphor are negligible in this study, so the background noise in the case of the white-LED was much reduced in comparison with the SHG/YAG laser case [15]. The other problem is the scattering of the laser light and the Raman light in the resin. In the wavelength region used in this study $(633-657 \mathrm{~nm})$, it was measured that the laser light and the Raman scattering light were reduced to around $70 \%$ by the scatterer in the resin. These results suggest that junction temperature estimation for a white-LED is possible, although the Raman signal intensity becomes about half by $70 \%$ decreasing each of the laser beam and the Raman light. It should be noted that the Raman signal from a white-LED covered by the same resin was detected successfully in the recent experiment.

\section{Conclusions}

A pulsed-laser Raman scattering technique was applied to the temperature estimation of the junction of one of chips on a fluorescent-resin-less blue-LED package. It is shown that the Raman shift of $E_{2}^{\mathrm{H}}$ mode scattered from a GaN layer of the LED is dependent with the temperature, and that the junction temperature of the LED during the operation can be 
estimated by this method in spite of existence of intense stray light, Rayleigh scattering and LED emission light. By the appropriate selection of the incident laser wavelength, background noise, which is caused mainly by fluorescence emitted simultaneously with the Raman signal from the phosphor- and scatterer-mixed resin of a white-LED, can be removed. Therefore, the pulsed-laser Raman scattering method proposed in this study would be one of the prospective candidates of a remote and standard system for estimation of the junction temperature of white-LEDs during the operation.

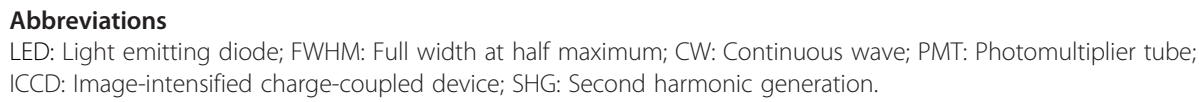

\section{Authors' contributions}

$M H, Y Y$ and $Y M$ piled argument up, then proposed the topic, conceived and designed the study as a result of the meeting. MH, YY and ST carried out the experimental study, and analyzed the data. KT and YM helped in the interpretation of experimental data. All authors read and approved the final manuscript.

\section{Acknowledgements}

This work was supported by JSPS KAKENHI Grant Number 26420237 partially.

\section{Author details}

${ }^{1}$ Eco Solutions Company, Panasonic Corporation, 1048 Kadoma, Kadoma City, Osaka 571-8686, Japan. ${ }^{2}$ Interdisciplinary Graduate School of Engineering Sciences, Kyushu University, 6-1 Kasugakoen, Kasuga City, Fukuoka 816-8580, Japan.

Received: 29 November 2014 Accepted: 9 July 2015

Published online: 22 July 2015

\section{References}

1. Ahmad I, Holtz M, Faleev NN, Temkin H. Dependence of the stress-temperature coefficient on dislocation density in epitaxial GaN grown on a-Al $\mathrm{O}_{3}$ and 6H-SiC substrates. J Appl Phys. 2004;95:1692.

2. Ahmad I, Kasisomayajula V, Song DY, Tian L, Berg JM, Holtz M. Self-heating in a GaN based heterostructure field effect transistor: Ultraviolet and visible Raman measurements and simulations. J Appl Phys. 2006;100:113718.

3. Fukuyama T, Kai M, Manabe Y, Yamagata Y, Tomita K. Proceedings of the 66th Joint conference of Electrical, Electronics and Information Engineers in Kyushu. Kumamoto: Kumamoto University; 2013. p. 24-5.

4. Hwang WJ, Lee TH, Kim L, Shin MW. Determination of junction temperature and thermal resistance in the GaN-based LEDs using direct temperature measurement. Phys Stat Sol (c). 2004;1:2429-32.

5. Kuball M, Pomeroy JW, Rajasingam S, Sarua A, Uren MJ, Martin T, et al. High spatial resolution micro-Raman temperature measurements of nitride devices (FETs and light emitters). Phys Stat Sol (a). 2005;202:824-31.

6. Kuball M, Riedel GJ, Pomeroy JW, Sarua A, Uren MJ, Martin T, et al. Time-Resolved Temperature Measurement of AlGaN/GaN Electronic Devices Using Micro-Raman Spectroscopy. IEEE Electr Device Lett. 2007;28:86-9.

7. Liu MS, Bursill LA, Prawer S, Nugent KW. Temperature dependence of Raman scattering in single crystal GaN films. Appl Phys Lett. 1999;74:3125-7.

8. Murata S, Nakada H. Adding a heat bypass improves the thermal characteristics of a 50um spaced 8-beam laser diode array. J Appl Phys. 1992;72:2514-6.

9. Sarua A, Ji H, Kuball M, Uren MJ, Martin T, et al. Integrated Micro-Raman/Infrared Thermography Probe for Monitoring of Self-Heating in AIGaN/GaN Transistor Structures. IEEE Trans Electr Device. 2006;53:2438-47.

10. Senawiratne J, Li Y, Zhu M, Xia Y, Zhao W, Detchprohm T, et al. Junction Temperature Measurements and Thermal Modeling of GalnN/GaN Quantum Well Light-Emitting Diodes. J Electron Mater. 2008;37:607-10.

11. Song DY, Basavaraj M, Nikishin SA, Holtz M. The influence of phonons on the optical properties of GaN. J App Phys. 2006;100:113504

12. Wang Y, Xu H, SAlur S, Cheng AJ, Park M, Sakhawat S, et al. Determination of Junction Temperature of GaN-based Light Emitting Diodes. In: Electroluminescence and Micro-Raman Spectroscopy, editor. Proceedings of 2009 International Conference on Compound Semiconductor Manufacturing Technology. Tampa: Marriott Waterside; 2009. p. 18-21.

13. Xi Y, Schuberta EF. Junction-temperature measurement in GaN ultraviolet light-emitting diodes using diode forward voltage method. Appl Phys Lett. 2004;85:2163-5.

14. Xi Y, Xi JQ, Gessmann T, Shah JM, Kim JK, Schubert EF, et al. Junction and carrier temperature measurements in deep-ultraviolet light-emitting diodes using three different methods. Appl Phys Lett. 2005;86:031907.

15. Yamagata Y, Horiuchi M, Tomita K, Manabe Y. Proceedings of the 67th Joint conference of Electrical, Electronics and Information Engineers in Kyushu. Kagoshima: Kagoshima University; 2014. p. 18-9. 